\title{
Estudio sobre la gestión tecnológica y del conocimiento en una organización creadora de conocimiento
}

\author{
Navarro de G., Ketty ${ }^{*}$ \\ Romero de G., Eunice** \\ Bauza, Roberto*** \\ Granadillo, Víctor A. $^{\star \star \star \star}$
}

\section{Resumen}

En el presente artículo se exponen los resultados de un trabajo exploratorio, de carácter cualitativo, efectuado en un laboratorio de investigación de la Facultad Experimental de Ciencias de la Universidad del Zulia. Concretamente, el trabajo se levantó para cubrir dos objetivos específicos:1) analizar el proceso de gestión del conocimiento en el Laboratorio de investigación Analítica (LIA) y, 2) Estudiar el proceso de gestión tecnológica en el LIA. Como metodología de trabajo fundamental se empleó la observación directa a la rutina diaria del proceso de creación de conocimiento, durante un período de tres meses y se aplicaron entrevistas no estructuradas a todos los integrantes del mismo. Los resultados conforman una primera aproximación al estudio formal del proceso de creación de conocimiento en grupos de investigación pertenecientes a instituciones de educación superior y, en tal sentido, evidenciaron algunos mecanismos que están incidiendo en los procesos de generación y movilización del conocimiento. También revelaron insumos experienciales para comprender el nuevo estatus de la tecnología en la generación de conocimientos.

Palabras clave: Gestión tecnológica, universidades, grupos de investigación, gestión del conocimiento, química analítica.

Recibido: 28-09-04 Aceptado: 17-5-06

* Socióloga, magíster en Planificación y Gerencia de Ciencia y Tecnología, integrante de la línea de investigación sobre gestión del conocimiento en la Maestría de Planificación y Gerencia de Ciencia y Tecnología, Departamento de Ciencias Humanas, Facultad Experimental de Ciencias de la Universidad del Zulia, Maracaibo, Venezuela. E-mail: kettydeg@cantv.net

** Filósofa. Profesora titular del Departamento de Ciencias Humanas, Facultad Experimental de Ciencias de la Universidad del Zulia, Maracaibo, Venezuela.

*** Químico, Profesor-Investigador adscrito al Laboratorio de Petroquímica y Surfactantes Departamento de Química, Facultad Experimental de Ciencias de La Universidad del Zulia, Maracaibo, Venezuela.

**** Químico, Profesor-Investigador adscrito al Laboratorio de Instrumentación Analítica, Departamento de Química, Facultad Experimental de Ciencias de la Universidad del Zulia, Maracaibo, Venezuela. E-mail: vkgranadillo@cantv.net 


\title{
A Study of the Management of Technology and Knowledge in a Knowledge-Creating Organization
}

\begin{abstract}
The present article explains the results of exploratory work of a qualitative nature carried out in a research laboratory of the Experimental Faculty of Sciences at the University of Zulia. The work was carried out with two specific objectives: 1) to analyze the process of knowledge management in the LAI (Laboratory of Analytical Instrumentation) and, 2) to study the process of technological management in the LAI. As a fundamental work methodology, direct observation of the daily routine of the process of creating knowledge was used during a period of three months and non-structured interviews were applied to all members of the study. The results offered an initial approximation to a formal study of the process of creating knowledge in research groups belonging to institutions of higher learning, and in this sense, evidenced some mechanisms that have a bearing on the processes of generating and mobilizing knowledge. Experiential input for understanding the new status of technology in the generation of knowledge was also revealed.
\end{abstract}

Key words: Management of technology, universities, research groups, management of knowledge, analytic chemistry.

\section{Introducción}

En el presente trabajo se exponen los resultados de un ejercicio que nos propusimos en el Laboratorio de Cambio Tecnológico, de la Maestría en Planificación y Gerencia de Ciencia y Tecnología de la Facultad Experimental de Ciencias de LUZ, con el propósito de discutir cómo afecta el cambio tecnológico a la dinámica interna de las organizaciones generadoras de conocimientos. Concretamente el trabajo se levantó para cubrir dos objetivos específicos: 1) analizar el proceso de gestión del conocimiento en el LIA y, 2) Estudiar el proceso de gestión tecnológica en el LIA.

El trabajo práctico se desarrolló en el Laboratorio de Instrumentación Analítica de la Facultad Experimental de Ciencias de la Universidad del Zulia, Venezuela. Su escogencia se debió a su aper- tura hacia el análisis interno de su institución, su sentido de la organización, y por su trayectoria científica, evidenciada en las numerosas publicaciones, personal de investigación formado; y sobre todo, por el impacto de algunas de sus investigaciones en el entorno social y político de la región.

Como metodología de trabajo fundamental se empleó la observación directa a la rutina diaria del proceso de creación de conocimiento en el mencionado laboratorio, durante un período de tres meses y se aplicaron entrevistas no estructuradas a todos los integrantes del mismo: coordinador, profesores y estudiantes de pre y postgrado. Las entrevistas fueron grabadas y analizadas mediante el método de análisis fenomenológico.

Desde el punto de vista teórico se utilizó el enfoque de gestión tecnológica de Sumanth y Sumanth (1998) para definir el ciclo de adquisición tecnológica del 
laboratorio y el modelo de creación de conocimiento de Nonaka y Takeuchi (1995), para entender el comportamiento de sus integrantes durante el proceso de creación del conocimiento. Todo ello con el propósito de generar aportes técnicos y metodológicos que sirvan de base para futuros estudios relacionados con el proceso de creación de conocimiento en las universidades públicas nacionales, lo cual podría ser de gran utilidad para determinar factores determinantes de niveles de productividad de sus grupos de investigación.

El corazón del trabajo está estructurado en cinco partes: en la primera se exponen los fundamentos teóricos que enmarcan el análisis. En la segunda, se realiza una descripción de la actividad académica que se desarrolla en el LIA, evidenciándose algunos elementos de su rutina de creación de conocimiento. En la que se realiza una revisión acerca de la evolución de la química analítica como ciencia y técnica, con la finalidad de ubicar el conocimiento del LIA con respecto al avance de la química analítica como ciencia técnica hoy. En la cuarta parte se describen los resultados de los procesos de gestión tecnológica y de gestión del conocimiento, evidenciándose que para el grupo de investigación, lo primordial es la creación de conocimiento, función dentro de la cual se inserta la gestión de la tecnología. Finalmente, en las conclusiones se realizan algunos comentarios y recomendaciones para la ampliación del marco competitivo de esta organización y el abordaje teórico-metodológico de futuras investigaciones.

\section{Una visión panorámica de la gestión tecnológica}

De manera creciente se destaca la importancia de las innovaciones incrementales, fruto de procesos de aprendizaje tecnológico, como motor de los altos ritmos de innovatividad de las empresas. Con ello el modelo lineal de innovación ha ido cediendo paso a modelos de carácter más iterativo (Freeman, 1982; Hippel, 1988; Rothwell, 1994). Ese cambio ha entrañado un nuevo enfoque en el tipo de información que se debe acopiar para analizar el fenómeno innovador. Se parte de la consideración de que muchas de las actividades vinculadas al proceso innovador son intangibles y como tales son difíciles de medir con las cuentas nacionales, las estadísticas convencionales de desempeño en ID o las tasas de patentamiento (Dutrénit y Vera-Cruz, 2000). Las tecnologías que conforman este nuevo sistema son esencialmente tecnologías basadas en la valorización de la información, a tal punto que tiene por lógica sustituir a la materia o la energía por información, lo que constituye una ruptura radical con los anteriores sistemas, que tenían por lógica la sustitución de la fuerza humana por la energía artificial y la materia bruta o natural por la materia transformada (Smaïl, 1990: 124), convirtiéndose así la información en uno de lo mejores soportes para la competitividad de la empresa, en conjunto con los conocimientos de las personas. 
Este proceso es uno de los efectos de la mutación tecnológica, que ha significado también cambios radicales en las formas de organización de la empresa, modificando las formas de organización y los hábitos de trabajo. Las nuevas formas de gestión, llamadas "inteligentes", están condicionados por la expresión de reconocimiento del grupo, de su capacidad de iniciativa y de inteligencia que deben presidir la implantación, el desarrollo y la eficacia de la nueva base tecnológica de la actividad (Smaïl, op cit: 227). Esto significa tomar decisiones inteligentes y a tiempo de la tecnología requerida, es decir, "gestionar la tecnología".

Sumanth y Sumanth (citado en Gaynor, 1998) definen a la gestión de tecnología como el proceso mediante el cual una organización se percata de la existencia de una tecnología, la adquiere, la adapta a sus necesidades, obtiene avances en la misma y la abandona para procurar otra que mejor satisfaga sus necesidades y así incrementar o mantener su productividad, definición que compartimos para efectos del presente trabajo. Este proceso es descrito como un ciclo continuo que puede ser aplicado a cualquier nivel dado: producto, servicio, función, centro de trabajo, planta/división, corporación e industria nacional o internacional. Este ciclo consta de cuatro fases:

La fase de percepción es la primera fase del ciclo de la tecnología, en la cual una empresa posee un mecanismo formal para llegar a ser conscientes de la existencia de tecnologías emergentes relevantes a sus necesidades. Algunas empresas forman "grupos de investigación" interdisciplinaria que recopilan la información por cualquier medio. La fase de adquisición involucra la adquisición real de una tecnología particular. Para ir de la fase de percepción a la fase de adquisición, es necesario elaborar un estudio de factibilidad técnica y económica, antes de justificar y adquirir una tecnología. La fase de adaptación virtualmente, toda empresa termina adaptando una tecnología adquirida para sus necesidades particulares, para los efectos, es necesario dedicar tiempo y esfuerzo al estudio de la importancia de una tecnología particular a las necesidades de la empresa. La fase de avance: cuando el capital es limitado, no se puede adquirir y abandonar tecnología en forma indiscriminada, por lo que se hace imperativo improvisar las tecnologías adquiridas adaptándolas a las necesidades propias de la empresa. Por último, la fase de abandono es la fase más crítica debido a que en ella se toman las decisiones con respecto a la obsolescencia de una tecnología en particular. Ante la rápida obsolescencia de las tecnologías existentes (basadas en el producto, proceso, en la información y en la gestión) el momento oportuno para introducir nuevas tecnologías es crítico para ganar en el juego de los negocios sin mencionar su supervivencia.

En consecuencia, el conocimiento de las tecnologías sobre las que se quiere actuar obliga a saber el nivel de avance del conocimiento tecnológico de la rama en la que se desenvuelve el proyecto, así como dónde se puede acceder a estas tecnologías de la manera más eficiente posible. Asimismo, la adecuada gestión del recurso tecnológico se apoya en la existencia de personal cualificado para esas actividades. El conocimiento de los expertos existentes en la organización 
sobre diferentes tecnologías, así como de la experiencia de la organización en su conjunto, es un recurso potencial que toda la organización debe saber gestionar y que forma parte de la "gestión del conocimiento". Así, los procesos relacionados con la gestión del conocimiento se superponen a la de desarrollo de los proyectos y pueden considerarse parte de los relacionados con la gestión del recurso tecnológico (Hidalgo, et al: 2002:38).

\section{La productividad sustentada en el uso del conocimiento:}

En la gestión tecnológica, el uso de los conocimientos es fundamental y esto se justifica en el hecho de que en el reacomodo económico de este tercer sistema, con sus fuertes implicaciones sociales y políticas, se plantea una nueva estrategia de competencia, marcada por las capacidades para producir, circular y utilizar conocimientos en la producción de bienes y servicios y en los procesos de toma de decisiones; tanto así que reduce significativamente la importancia relativa de los recursos naturales y de la energía en el desarrollo económico y social de las naciones.

De acuerdo con los nuevos enfoques de la organización empresarial, los recursos naturales (su abundancia o escasez), han perdido gran parte de su capacidad para explicar las disparidades de productividad y de crecimiento entre los países. En cambio, la mejora de la calidad del equipo físico y del capital humano, es decir, de sus recursos y capacidades, actualmente son las variables que mejor explican el crecimiento económico. En otras palabras, la creación de nuevos conocimientos y de nuevas ideas y su incorporación al equipo físico y a las personas representan el capital intangible decisivo en los niveles de productividad y competitividad de las empresas.

Este capital intangible está constituido por inversiones en capacitación, instrucción, actividades de I y D, información y coordinación; es decir, por inversiones consagradas a la producción y a la transmisión del conocimiento. La otra gran partida del capital intangible corresponde a los gastos en salud; en otras palabras, a inversiones que mejoran las características físicas del capital humano (David y Foray (2002). Así, se afirma que en el paradigma económico de hoy, en el cual en la incertidumbre es la única certeza, una fuente segura y duradera de ventaja competitiva es el conocimiento. Las compañías acertadas son las que crean constantemente nuevo conocimiento, lo diseminan extensamente a través de la organización, y lo incorporan rápidamente a nuevas tecnologías y productos (Nonaka, 1991).

En función de esta premisa, algunos autores han dedicado numerosos estudios para explicar y generar fórmulas para gestionar el conocimiento, generando nuevos conceptos y nuevas herramientas para el análisis, dando origen a la teoría de la gestión del conocimiento y los modelos para la creación de conocimientos. En este sentido, destacan los aportes de Hansen (1999), Zollo y Winter (1998), Thomas Davenport (1995), Nonaka y Takeuchi (1995), Hedlund (1994), Kogut y Zander (1992), entre otros.

De todos ellos, el modelo de Nonaka y Takeuchi (1995) es el más conocido y el cual se ha tomado como refe- 
rencia para nuestro análisis. En este modelo se presta mucha atención al proceso de creación de conocimiento y empieza distinguiendo dos dimensiones: Una dimensión epistemológica: en el cual se distinguen dos tipos de conocimiento: el explícito y el tácito. El conocimiento explícito es el que está expresado de manera formal y sistemática, además, puede ser comunicado fácilmente y compartido en forma de unas especificaciones de producto, una fórmula científica o un programa de ordenador. Es aquel conocimiento que puede codificarse. El conocimiento tácito resulta difícil de expresar formalmente y por tanto es difícil de comunicarlo a los demás. Este tipo de conocimiento está profundamente enraizado en la acción y en el cometido personal dentro de un determinado contexto. Se señalan cuatro posibles modos de conversión entre los dos tipos de conocimiento:socialización (conversión de conocimiento tácito en tácito), externalización (conversión de conocimiento tácito en explícito) internalización (conversión de conocimiento explícito en tácito) y combinación (conversión de conocimiento explícito en explícito); y una dimensión ontológica: en la cual se distinguen cuatro niveles de agentes de creadores del conocimiento: el individuo, el grupo, la organización y el nivel interorganizativo. De acuerdo con este modelo, el conocimiento se crea siempre en el individuo, pero ese conocimiento individual se transforma en conocimiento organizativo valioso para toda la empresa.

\section{EI LIA como organización:}

El Laboratorio de Instrumentación Analítica (LIA) tiene su origen en las políticas académicas del Departamento de Química de la Facultad Experimental de Ciencias de la Universidad del Zulia (LUZ), de finales de la década de los años 70 , destinadas a formar personal especializado en química analítica, por lo cual decide enviar a un profesor a realizar un doctorado en Química Analítica en la Universidad de Manchester, Inglaterra. Es así como el 23 enero de 1983 este laboratorio inicia sus actividades, dirigido por el primer Ph.D en química del Departamento. El 23 de septiembre de 1983 adquiere un espacio físico propio, en las mismas instalaciones en donde funcionaba el Laboratorio de Investigaciones Químicas, que era muy general y básicamente servía para apoyar las tesis de grado de los primeros egresados del Departamento (LIA, 1995). EI LIA, entonces, fue el primer laboratorio del Departamento de Química de LUZ, organizado sistemáticamente en líneas de investigación, proyectos y programas, con objetivos y visión y misión definidas, aunque estas últimas nunca fueron escritas. En este sentido, a continuación se presenta lo que se asume como la misión y visión en la cultura organizacional de este Laboratorio.

- Misión: EI LIA es un laboratorio de investigación que tiene como misión realizar estudios de interés para la región, así como apoyar a estudiantes y profesores del Departamento de Quí- 
mica para realizar investigaciones en el área.

- Visión: Llegar a convertirse en un centro de excelencia nacional e internacional, escenario de estudiantes y profesores de LUZ, para realizar investigaciones y tesis en el área, financiados por CONICIT, CONDES y otras organizaciones nacionales e internacionales que brinden apoyo a la investigación.

Aunque no es nuestra intención analizar el tipo de gerencia con la cual ha funcionado el LIA, es importante aclarar algunos aspectos que pueden servir para comprender su situación actual y además puedan ser de interés para futuros estudios. Su historia podría dividirse en dos etapas: La primera que arranca desde su fundación hasta 1995, a la cual corresponde la mayor parte de su producción académica, sobre todo, en lo referido a las publicaciones y presentaciones en congresos, correspondiéndole el 93.4\% de las publicaciones generadas, con un promedio de 6 publicaciones al año (lo cual es un número elevadísimo, según las normas internacionales y venezolanas que exigen al menos una (1) publicación cada dos (2) años). Igualmente, corresponde el $100 \%$ de las presentaciones en congresos internacionales y el $50 \%$ de las presentaciones en congresos nacionales. En esta etapa se adquirieron todos los equipos que existen actualmente en el laboratorio, teniendo acceso a la tecnología de análisis instrumental de vanguardia para la época. En efecto, el LIA fue el primer laboratorio de LUZ que adquirió un espectofotómetro de absorción atómica, por ejemplo. Todo ello gracias a la bonanza económica que gozaba la Universidad del Zulia y el país en general, lo cual facilitaba el proceso de adquisición.

Este periodo se caracteriza por un liderazgo controlador y autoritario, en donde todas las decisiones y actividades se concentraban alrededor del líder. Su estructura organizativa era de tipo vertical sin líneas de mando intermedias. Se utilizaba la política del castigo-premio que obligaba a sus integrantes a trabajar hasta altas horas de la noche, días feriados y fines de semana, lo cual se "premiaba" con viajes al exterior para presentar los resultados de las investigaciones en eventos científicos. Esto les permitía estar actualizados en los avances de los conocimientos científicos y tecnológicos en el área, y además, establecer relaciones con investigadores y centros de excelencia mundial que en algunas ocasiones se consolidaban en trabajos conjuntos que generaban publicaciones

La segunda etapa que se inicia en 1996 hasta el presente; se caracteriza por una producción moderada de artículos científicos (una (1) por año), la carencia de presentaciones en congresos internacionales y la escasez de financiamiento para el desarrollo de los proyectos y las actividades en general; sin embargo, sigue manteniéndose en los estándares internacionales de producción. La escasez de recursos actuales obedece a la profundización de la crisis universitaria, reflejo de la del país, que ocasiona una merma considerable en los recursos destinados a la investigación. Esta situación coincide con la tesis de Smaïl Aït-et El Hadj del retroceso general y regular de los gastos para investigación y desarrollo y en especial, la tasa de expansión de la investigación universitaria, durante el 
proceso de cambio del segundo al tercer sistema tecnológico.

Entre las contribuciones más importantes de este Laboratorio para la Universidad del Zulia y la región zuliana en general, se pueden mencionar: es referencia nacional e internacional en el desarrollo de métodos para determinar presencia de metales tóxicos en seres humanos y alimentos. Sus trabajos han realizado aportes importantes al conocimiento de cómo está afectando la contaminación ambiental generada por la industria petrolera, a la población de Maracaibo y sus alrededores (LIA, 2001). Su competitividad está basada en la generación de conocimientos que causen gran impacto social.

\section{Evolución de la química analítica como ciencia y técnica o el nuevo estatus de la tecnología en la evolución de la química analítica}

Este apartado se incluye con la finalidad de mostrar el cambio tecnológico ocurrido en la química analítica durante los últimos años y la necesidad permanente de gestión tecnológica que requiere el LIA. En este sentido, según los expertos en el área la química analítica ha evolucionado mucho en los últimos años, actualmente, la tecnología gira en torno a reducir los procesos y el tiempo para el tratamiento de las muestras, en un $80 \%$ con respecto a los métodos tradicionales.

De acuerdo con el Doctor Roberto Bauza (2002) para entender un poco el estado actual de la química analítica como ciencia, es necesario hacer referencia a su evolución. La primera época de la evolución de la química analítica basada en una clasificación tecnológica, ocurre desde los albores de principio del siglo XX hasta 1955, en lo que se conoce como el aumento de los diseños de instrumentación (en este período se inventó el espectrofotómeto de absorción atómica). Este período algunos autores lo han clasificado como la época clásica. Posteriormente, viene la época moderna que algunos autores la han fijado hasta finales del 75, cuando se introducen los procesos de automatización y aparecen las técnicas que se denominan informáticas, comienzan los famosos softwares que inician el proceso de automatización. Acá, la calidad pasa a ser de no sistemática a sistemática; es decir, se comienza a pensar seriamente en los parámetros de calidad. En la época contemporánea comienza el proceso de miniaturización, en la se crean las técnicas "liliputienses" denominadas hoy día "lab on a chip", o el laboratorio en un chip. Un aporte de las técnicas liliputienses a la investigación analítica es, por ejemplo, que a través de los chips se puede mineralizar dilusiones a nivel de un compuesto.

Desde el punto de vista conceptual la química analítica ha evolucionado procurando la mejora de las propiedades analíticas, la exactitud, la cual ahora se mide en nanounidedes $\left(10^{-9}\right.$.) ó $(0,000000001)$ Las terminologías también han avanzado hacia los enfoques metrológicos, por ejemplo, la exactitud se llama ahora trazabilidad, y la precisión ahora se enfoca en términos de incertidumbre etadística (Bauza, 2002). Resumiendo, las tendencias actuales de la química analítica están en el desarrollo de calidad, la ampliación de fronteras, la simplificación, la 
automatización, la miniaturización y los sistemas analíticos de respuestas rápidas (Varcalcel 1999).

\section{Resultado acerca del proceso de gestión tecnológica en el LIA}

En los grupos de investigación académicos, más que gestionar la tecnología, lo que importa es gestionar la adquisición de nuevos conocimientos, por cuanto la adquisición de la tecnología forma parte del proceso mismo de creación del conocimiento. En este sentido, partiendo de la fases del ciclo de la tecnología de David J Sunmanth y John J. Sumanth, $y$ tomando como referencia la experiencia propia con la gerencia de ciencia y tecnología en centros y laboratorios de investigación de la Universidad del Zulia, intentamos describir como se gestiona la adquisición de nuevas tecnologías para la creación de nuevos conocimientos en una organización generadora de conocimientos, haciendo alusión específica al Laboratorio de Instrumentación Analítica (LIA) de la Facultad Experimental de Ciencias, por ser la unidad de estudio del presente trabajo:

Fase de percepción: A diferencia de cómo se desarrolla esta fase en el sector empresarial, en el laboratorio en cuestión, el proceso es diferente, no necesita conformar grupos para esta tarea, en virtud de que por naturaleza los investigadores adscritos están en permanente búsqueda tanto de nuevas tecnologías como técnicas que le permitan avanzar en el proceso de creación de conocimiento. La manera más común de conocerlos es a través de la asistencia a congresos y otros eventos nacionales e internacionales, la asistencia a exposiciones internacionales de tecnología que organizan los fabricantes, los contactos y confrontaciones con sus pares en el mundo, por consultas en la Internet, por la revisión de artículos científicos, por el recibo de catálogos desde las empresas fabricantes o vendedoras, o por la experiencia de colegas en programas de intercambio o estudios de doctorado en universidades nacionales y extranjeras, entre otros.

Fase de adquisición: En el LIA, al igual que el resto de los laboratorios de LUZ la adquisición de nueva tecnología se justifica mediante los proyectos de investigación, que son su quehacer diario y allí se establece su factibilidad técnica y económica. La limitante fundamental está en la carencia de recursos económicos para obtenerla, lo cual es difícil de solventar por ser un problema estructural. Como alternativa desde su fundación, pero notándose un incremento importante durante los últimos diez años, se recurre a la donación por parte de organismos públicos y privados del país y al trabajo colaborativo con otras facultades de LUZ o instituciones de la región.

Fase de adaptación: En el LIA este proceso es natural, los investigadores son los primeros promotores de la importancia de la tecnología para su competitividad como creadora del conocimiento. El estudio sistemático de los nuevos adelantos científicos y tecnológicos en el mundo y el desarrollo permanente de proyectos de investigación les da seguridad a la hora de adquirir la tecnología.

Fase de avance: En el LIA no se realizan adaptaciones en equipos cuya tecnología se considera obsoleta porque 
no cuentan con personal para tal fin, es posible que desde el punto de vista técnico se realicen algunos cambios que optimicen el uso del mismo como por ejemplo, pequeñas adaptaciones. Lo que sí se observó fue un esmerado cuidado en la conservación y mantenimiento de los equipos, pues según sus argumentos "les cuesta mucho obtener el financiamiento para repararlos".

Fase de abandono: En el LIA están concientes de esto y en este caso el problema es de recursos económicos y no de conocimientos. Por lo general, la tecnología se sigue utilizando tal cual, hasta que se pueda adquirir la más avanzada, lo cual siempre estará sujeto a la disponibilidad financiera del donante 0 del organismo financiero del proyecto en donde se incluya su adquisición.

En función de esta descripción, se podría concluir de manera parcial que en el LIA, el ciclo de la tecnología es diferente al de las organizaciones empresariales y el esquema de los autores mencionados no se adapta literalmente al análisis de su proceso de gestión de tecnología. En este sentido, que este proceso se podría esquematizar en el diagrama 1 de la manera siguiente:

Describiendo el diagrama 1, el ciclo se inicia cuando los líderes de líneas de investigación se percatan de las innovaciones que han surgido en el marco de la

\section{Diagrama 1 \\ Ciclo de la tecnología en el laboratorio de instrumentación analítica de la facultad experimental de ciencias}

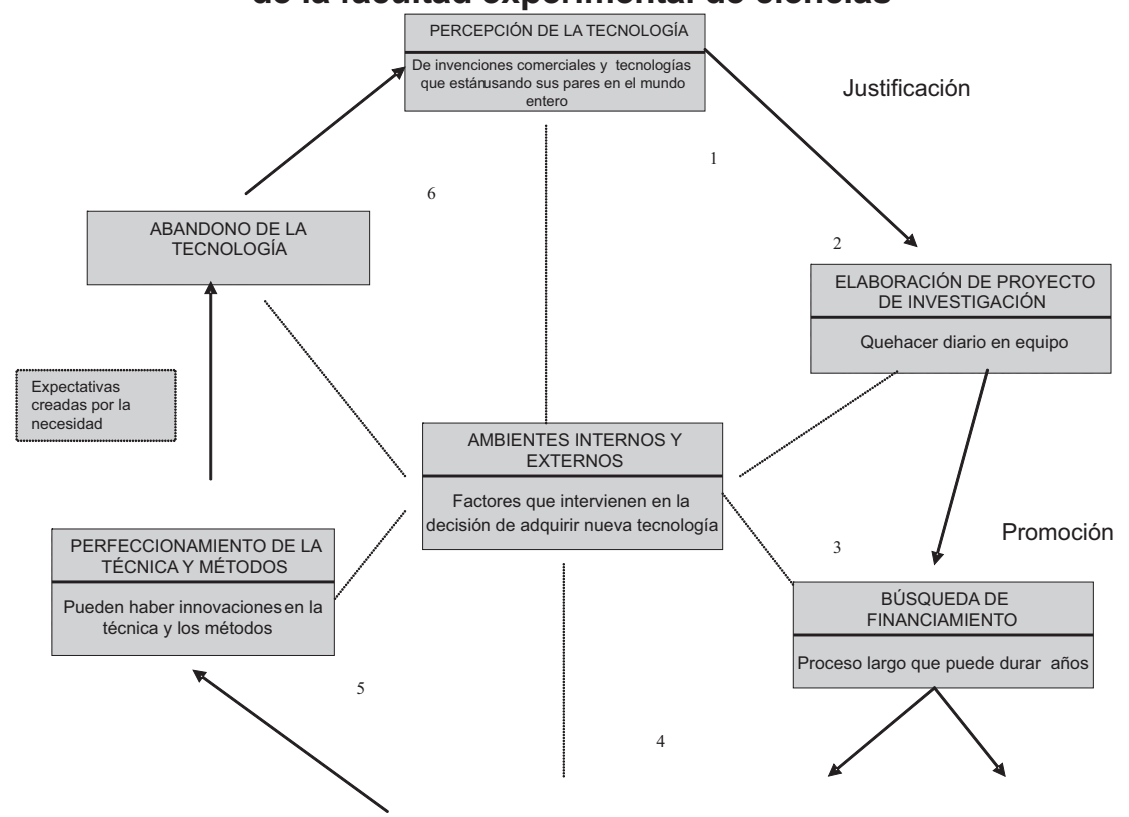

Fuente: Elaboración propia. 
tecnología fundamental para el desarrollo de sus investigaciones, innovaciones que por lo general establecen la inclusión de nuevos conceptos y métodos que a su vez determinan la competencia de los resultados (fase 1). Esta nueva tecnología se plantea como una necesidad para el desarrollo de las nuevas investigaciones que tienen en mente, por lo cual se diseñan uno o varios proyectos de investigación (fase 2), este (os) proyecto (s) sirve(n) de base para promocionar la nueva tecnología ante las autoridades de la universidad y el ente financiero.

El proyecto elaborado se somete a la consideración de uno o más organismos de financiamiento (cofinanciamiento), proceso que muchas veces se convierte en un laberinto de procedimientos $y$ de idas y venidas que producen desgaste y tensión. La duración de esta etapa es incierta, pues la respuesta puede tardar años. (fase 3). Sí la respuesta es negativa se continúa con el mismo equipo hasta obtener el financiamiento. Si el proyecto se aprueba, nuevamente es preciso someterse a procedimientos institucionales para su adquisición (fase 4). Cuando la tecnología es desconocida para los miembros del grupo, lo más natural es que alguno de sus miembros realice un entrenamiento, para luego servir de agente multiplicador. En la rutina de investigación se perfecciona el uso y manejo del equipo, así como de la tecnología en su conjunto (fase 5). En este proceso se plantean nuevas interrogantes y necesidades que conllevan a plantear nuevas investigaciones, el avance de métodos y también el abandono de nuevas tecnologías (fase 6). A manera de ejemplo de esta fase en los actuales momentos el la- boratorio se encuentra en una fase de abandono de la tecnología relacionada con los espectrofotómetros de absorción atómica por la de electroforesis capilar. En la primera, la unidad de volumen son mililitros $\left(10^{-3}\right)$ y en el segundo, los volúmenes de muestra pueden ser representados en nanolitros $\left(10^{-9}\right)$, ejemplo claro de la tendencia hacia la miniaturización.

De la interpretación del Diagrama 1 también se desprende que obviamente el tiempo de respuesta para la presentación de resultados es más largo en el LIA que en la empresa, por cuanto entre las fases 3 y 4 podrían darse márgenes de tiempo de hasta tres años, según el ente financiador del proyecto de investigación. También este tiempo de respuesta está directa y simultáneamente relacionado con la naturaleza de su función como organización generadora de conocimientos. Así, tanto la gestión tecnológica como la gestión de conocimiento inherentes a la empresa generan resultados que son de aplicación inmediata para la propia organización, de acuerdo a los requerimientos de rápido retorno de sus tasas de inversión. Por el contrario, en los grupos de investigación de las universidades, estos procesos son más lentos y la velocidad de respuesta menos rápida, puesto que no obedece a presiones de índole financiero-productivas.

\section{Resultados en relación al proceso de Gestión de Conocimientos en el LIA}

De acuerdo con el modelo de Nonaka y Takeuchi, las cuatro formas de conversión del conocimiento surgen cuando el conocimiento tácito y el explíci- 
to interactúan, y en este proceso dinámico de creación de conocimiento se genera un ciclo continuo y acumulativo de generación, codificación y transferencia del conocimiento, la llamada espiral de creación del conocimiento. Al igual que en las comunidades de conocimiento observadas por los mencionados autores, este proceso se desarrolla de manera similar en el LIA.

La dimensión epistemológica está representada por los constructos teóricos que enmarcan el saber de la química analítica, en ésta, el conocimiento explícito es expresado por lo productos que se generan, tales como proyectos de investigación, nuevas técnicas, nuevas metodologías, publicaciones y/o patentes, entre otros; como se sabe, es un conocimiento fácil de codificar, fácil de transmitir y fácil de entender. El conocimiento tácito está expresado por un cúmulo de conocimientos difíciles de expresar y transmitir y profundamente arraigado en su modelo mental. Acá se incluye no solamente la experiencia individual de cada investigador sino su visión acerca del conocimiento que crean.

En el marco de la dimensión ontológica, el individuo, el grupo, la organización y las relaciones interorganizativas forman un engranaje de difícil descomposición porque cada individuo forma al LIA y el LIA es el grupo y a la vez es la organización. Dentro de sus valores organizacionales, pertenecer al LIA es ser "liísta" y el que entra no solamente lo acepta, también debe hacerse partícipe de un proceso de aprendizaje de rutinas de creación de conocimiento que forman parte del conocimiento tácito acumulado durante toda la existen- cia del laboratorio. El calificativo "lísta" traduce un profundo arraigo a la organización, quedando excluido aquél que no lo sienta. Cuando el grupo se autodenomina "liísta" está compartiendo una misma visión y está construyendo su "ba", concepto que introduce Nonaka para definir el lugar o espacio mental y físico donde se crea conocimiento (Nonaka, 1991).

Durante el desarrollo del presente trabajo se observaron algunas situaciones que verifican lo dicho anteriormente:

- Para ingresar en el laboratorio se debe demostrar interés y vocación hacia la investigación y estar dispuesto a someterse a los procesos de aprendizaje.

- Para permanecer en el laboratorio, es necesario estar activo en por lo menos un trabajo de investigación, ser consecuente y demostrar responsabilidad.

- Si un trabajo es abandonado por el responsable, el grupo lo retoma para no perder la información ni el esfuerzo, en este caso se observó la iniciativa de los estudiantes para realizar esto.

- Se observa cierta independencia y autonomía en cuanto a las decisiones en torno a la parte experimental de los proyectos. Por ejemplo, los estudiantes se planifican para el desarrollo de los experimentos. Sí se daña un equipo, ellos mismos llaman a los técnicos o compran las piezas, en el caso de que esto no implique mucho dinero y puedan costearlo con los recursos del LIA (caja chica con aportes personalizados e individuales de estudiantes y profesores).

- Cada quien tiene asignada sus responsabilidades y da cuenta de ello en 
las reuniones semanales del grupo, es decir, los seminarios.

- Tienen libertad para establecer fechas de avances o resultados, en función del tiempo reglamentario.

- En el sistema de valores se observa mucha identificación con el laboratorio, sentido de pertenencia, orgullo hacia lo que es y representa como organización. En varias oportunidades manifestaron que el LIA es el mejor laboratorio en química clínica del país y que en el exterior son muy respetados.

- El ambiente observado es de alegría, camaradería, respeto, colaboración y solidaridad.

- Al inicio de cada sesión diaria (montaje de experimentos, arranque de equipos) y por medidas de seguridad y gestión de la caldad analítica, es obligatorio limpiar el laboratorio. Esto es realizado a diario por los estudiantes (en equipo), debido a que el obrero asignado por la Facultad, sólo asiste una vez por semana. Cada estudiante, al igual que el obrero, es entrenado para realizar la limpieza, la cual requiere de técnicas especiales.

En cuanto al modo de producir conocimiento explícito, los mecanismos del LIA son similares al de la mayoría de los grupos de investigación formales: el desarrollo de proyectos de investigación, la composición de artículos científicos, la confrontación con pares en congresos nacionales e internacionales, el dictado de clases, charlas, conferencias, todo ello utilizando las herramientas de las tecnologías modernas de información.

Una de las mayores evidencias de la formalidad del grupo para la creación de conocimiento es la realización de las reuniones o seminarios semanales de trabajo. En este seminario se presentan los avances individuales y colectivos del proceso de investigación, se aclaran dudas, se responden preguntas, se planifican actividades, se establecen estrategias, se corrigen errores, pero también las reuniones sirven para resolver conflictos entre el grupo, tratar problemas individuales, establecer estrategias de ayuda y estrechar los nexos entre los integrantes. Por lo general, el trato entre los integrantes de un grupo de investigación es familiar, su contacto va más allá de la rutina del trabajo y muchas veces se extiende al campo familiar. En las conversaciones que se establecen en dichas reuniones se confirma y reconfirma la pericia de los integrantes sobre su línea de trabajo, lo que permite la eficaz resolución de problemas.

Esto coincide con el proceso de socialización descrito por Nonaka (1998), el cual involucra el compartir, mediante la proximidad física, es decir, nuestro conocimiento tácito con otros individuos. Mediante la socialización, el individuo libera su conocimiento tácito para capturar el conocimiento tácito de los otros. Cuando se transfiere una imagen o idea personal directamente al colega o subordinado, se establece un lugar común para la creación de conocimiento.

\section{Conclusiones}

- Los resultados conforman una primera aproximación al estudio formal del proceso de creación de conocimiento en grupos de investigación pertenecientes a instituciones de educación 
superior y, en tal sentido, evidenciaron algunos mecanismos que están incidiendo en los procesos de generación y movilización del conocimiento. También revelaron insumos experienciales para comprender el nuevo estatus de la tecnología en la generación de conocimientos.

- De acuerdo con los planteamientos anteriores queda claro que en lugar de realizar gestión de tecnología, en el LIA es más importante gestionar los conocimientos y esto se convierte en fuerza vital para procurar cambios tecnológicos que incentiven la competitividad y el sostenimiento de dicha organización.

- A la luz de los cambios que han ocurrido en la química analítica durante los últimos años, se puede concluir que el proceso de creación de conocimiento en este laboratorio de la Universidad del Zulia, no solamente es afectado por la dinámica de la innovación en el área, sino que para generar conocimiento competitivo y globalizable, es necesario seguir la rutina de tales cambios.

- Como adelanto, desde el punto de vista organizacional, el LIA se ha ido adaptando a las tendencias gerenciales surgidas a partir de los cambios. Estas adaptaciones la realizan producto de la necesidad de sus rutinas y sin conciencia de los supuestos teóricos que subyacen en la implantación de nuevos esquemas gerenciales.

- El trabajo elaborado en el Laboratorio de Investigación Analítica permite comprobar la tesis de la mutación tecnológica de Smaïl, que señala cómo se adaptan las organizaciones al rea- comodo que supone el tercer sistema tecnológico. Es así como el LIA, en medio de la crisis científico-tecnológica más severas que ha vivido la Universidad del Zulia en los últimos años, ha tenido que adaptarse a los estilos organizacionales de vanguardia para garantizar su supervivencia y competitividad, esta última seriamente amenazada por la obsolescencia de la tecnología existente.

- En cuanto a las diferencias de la gestión tecnológica y la gestión del conocimiento se puede decir que los fundamentos de la gerencia del conocimiento toman en cuenta las condiciones que necesariamente deben darse para que el conocimiento se adquiera, se difunda, se utilice y se genere en la empresa, basándose en el proceso de aprendizaje y la innovación. Estos principios, frecuentemente se pasan por alto en la gestión de tecnología por creer que no son necesarios o por no entender su impacto en los resultados de la gestión.

- La gestión de tecnología se basa en la premisa de que la tecnología se vuelve obsoleta y debe ser reemplazada, con lo que lecciones aprendidas en el pasado, sencillamente se dejan de un lado. La gerencia del conocimiento pretende mantener y reutilizar el conocimiento adquirido sobre la base de que el conocimiento no se hace obsoleto.

- Por último, se recomienda profundizar este estudio y extenderlo hacia otros laboratorios de la Universidad, para tener un panorama más claro acerca de cómo se está preparando la universidad para adaptarse al ter- 
cer sistema tecnológico, sobre todo, porque actualmente se está gestando un proceso de transformación que pretende incorporar al conocimiento como eje fundamental de las actividades académico-administrativas de la misma, es de esperarse también, que este proceso de cambio lleve explícito el apoyo necesario para la adquisición, adaptación y asimilación de la tecnología básica para las investigaciones, las cuales, a nuestro modo de ver es pieza fundamental para establecer una sostenible estrategia competitiva en LUZ.

\section{Referencias Bibliográficas}

\section{Bauza, Roberto (2002). Las Nuevas Tenden- cias en la Química Analítica. Confe- rencia dictada el 27-09-2002, en el marco del XIX Aniversario del Labora- torio de Instrumentación Analítica de la Facultad Experimental de Ciencias de la Universidad del Zulia.}

David, Paul A.; Foray, Dominique (2002). Una introducción a la economía y a la sociedad del saber. Revista Internacional de Ciencias Sociales № 171.

Dutrénit, G.; Vera-Cruz, A. (2000). Fuentes de conocimiento para la innovación en la industria química mexicana. Comercio Exterior. México.

Gaynor, Gerard (1998). Manual de Gestión Tecnológica. Tomos I y II. Mc Graw Hill.
Hidalgo, Antonio; León, Gonzalo; Pavón, Julián (2002). La gestión de la Innovación y la Tecnología en las Organizaciones. Pirámide. Madrid, 2002

Laboratorio De Instrumentación Analítica (2001). Informe de Actividades 2001. Departamento de Química, Facultad Experimental de Ciencias. Universidad del Zulia.

Laboratorio De Instrumentación Analítica (1995): Propuesta de Conversión del LIA en Instituto de Investigaciones Analíticas. Departamento de Química, Facultad Experimental de Ciencias. Universidad del Zulia.

Nonaka, Ikujiro (1991). Knowledge-Creating Company. Harvard Business Review; Nov/Dec91, Vol. 69 Issue 6, p96, 9p, 3.

Nonakal, Ikujiro; konno; Noboru (1996). The Concept of ba. Building A Foundation For Knowledge Creation. California Management Review Vol 40, No, 3.

Nonaka, Ikujiro; Takeuchi, .Hirotaka (1995): The knowledge Creating Company. How Japanese Companies Create the Dynamics of Innovation. Oxford University Press.

Smaïl, Aït-El-Hadj (1990). Gestión de la Tecnología. Ediciones Gestión 3000, Barcelona, España.

Varcálcel, Miguel (1999). Principios de Química Analítica. Springer-Verlag. Barcelona, España. 